\title{
Response to: Comment on: "Do we have enough ophthalmologists to manage vision-threatening diabetic retinopathy? A global perspective"
}

\author{
Zhen Ling Teo ${ }^{1}$ Yih-Chung Tham ${ }^{1} \cdot$ Marco Yu $\mathbb{1}^{1} \cdot$ Ching-Yu Cheng $^{1,2,3} \cdot$ Tien Yin Wong ${ }^{1,2,3}$. \\ Charumathi Sabanayagam (i) ${ }^{1,2}$
}

Received: 7 April 2020 / Revised: 15 April 2020 / Accepted: 15 April 2020 / Published online: 27 April 2020

(c) The Royal College of Ophthalmologists 2020

\section{To the Editor:}

We thank Martins and colleagues for their letter regarding our study on the estimates of ophthalmologists manpower to tackle the burden of vision-threatening diabetic retinopathy (VTDR) worldwide [1]. We agree that training primary care physicians and optometrists and the use of technology for diabetic retinopathy (DR) screening can reduce DR-related blindness.

In our earlier publication [2], we emphasized the need for a paradigm shift in focus from tertiary to secondary and primary care to effectively tackle the burden of DR. Improving awareness and accessibility to DR screening and management is key. The Global Diabetic Retinopathy Advocacy Initiative group recommends appropriate task sharing and task shifting among ophthalmologists, primary care physicians and other healthcare professionals to deliver DR care in a collaborative and cost-effective manner [3]. An evidence-based shared eye care model in Singapore called the Primary Eye Care (PEC), demonstrated the effectiveness of task shifting [3] where by $80 \%$ of patients managed in tertiary hospitals were decanted to primary eye clinics run by trained general physicians and optometrists [3].

Telemedicine and virtual clinics can also aid in task shifting. In Singapore, under the Singapore Integrated

Charumathi Sabanayagam

charumathi.sabanayagam@seri.com.sg

1 Singapore National Eye Centre, Singapore, Singapore Eye Research Institute, Singapore, Singapore

2 Duke-NUS Medical School, Singapore, Singapore

3 Yong Loo Lin School of Medicine, National University of Singapore, Singapore, Singapore
Diabetic Retinopathy Programme (SiDRP), a telemedicinebased national screening programme for DR, digital retinal photographs taken at primary care settings are transferred for off-site assessment by trained graders, with an average reporting turnaround time of 30 minutes. Approximately only $10 \%$ of DR patients are referred to eye specialists, which significantly reduces unnecessary referrals [3]. In the UK, only $17.4 \%$ of patients attending virtual retina clinics were referred on to face-to-face clinics [4]. The success of telemedicine is important in settings with uneven or limited ophthalmologist resource. In India, teleconsultations in rural populations showed success, with majority of patients receiving treatment and $31 \%$ requiring referrals to tertiary centres [5]. Furthermore, artificial intelligence (AI)-based DR screening using deep-learning systems has the potential to be employed even in low-resource settings [6] such as Africa which has the lowest ophthalmologist density [1].

Overall, shifting and sharing of tasks, coupled with AI technology, could significantly reduce costs, increase access to quality care, and efficiency in DR care in the long run.

\section{Compliance with ethical standards}

Conflict of interest The authors declare that they have no conflict of interest.

Publisher's note Springer Nature remains neutral with regard to jurisdictional claims in published maps and institutional affiliations.

\section{References}

1. Teo ZL, Y-C Tham, Yu M, Cheng CY, Wong TY, Sabanayagam C. Do we have enough ophthalmologists to manage visionthreatening diabetic retinopathy? A global perspective. Eye. 2020. [Epub ahead of print]. 
2. Wong TY, Sabanayagam C. Strategies to tackle the global burden of diabetic retinopathy: from epidemiology to artificial intelligence. Ophthalmologica. 2020;243:9-20.

3. International Agency for the Prevention of Blindness. A global compendium on good practice: integrated care for diabetes and eye health [Internet]. 2018:69-72. https://www.iapb.org/tag/ compendium-of-good-practice/.

4. Kortuem K, Fasler K, Charnley A, Khambati H, Fasolo S, Katz M, et al. Implementation of medical retina virtual clinics in a tertiary eye care referral centre. Br J Ophthalmol. 2018;102:1391-5.
5. Das AV, Mididoddi S, Kammari P, Deepthi Davara N, Loomba A, Khanna R, et al. App-based tele ophthalmology: a novel method of rural eye care delivery connecting tertiary eye care center and vision centers in India. Int J Telemed Appl. 2019; 2019:8107064.

6. Ting DSW, Cheung CY, Lim G, Tan GSW, Quang ND, Gan A, et al. Development and validation of a deep learning system for diabetic retinopathy and related eye diseases using retinal images from multiethnic populations with diabetes. JAMA 2017; 318:2211-23. 